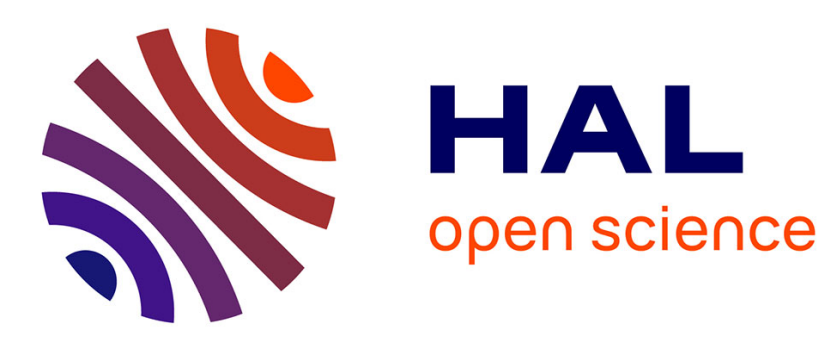

\title{
Behaviour of a solvent trapped in a physical molecular gel
}

I. Morfin, S. Spagnoli, C. Rambaud, S. Longeville, M. Plazanet

\section{To cite this version:}

I. Morfin, S. Spagnoli, C. Rambaud, S. Longeville, M. Plazanet. Behaviour of a solvent trapped in a physical molecular gel. Philosophical Magazine, 2016, 96 (7-9), pp.809-815. 10.1080/14786435.2015.1123821. hal-01583238

\section{HAL Id: hal-01583238 \\ https://hal.science/hal-01583238}

Submitted on 7 Sep 2017

HAL is a multi-disciplinary open access archive for the deposit and dissemination of scientific research documents, whether they are published or not. The documents may come from teaching and research institutions in France or abroad, or from public or private research centers.
L'archive ouverte pluridisciplinaire HAL, est destinée au dépôt et à la diffusion de documents scientifiques de niveau recherche, publiés ou non, émanant des établissements d'enseignement et de recherche français ou étrangers, des laboratoires publics ou privés. 
To appear in Philosophical Magazine

Vol. 00, No. 00, Month 20XX, 1-7

\title{
Behaviour of a solvent trapped in a physical molecular gel
}

\author{
I. Morfin ${ }^{\mathrm{a}}$, S. Spagnoli ${ }^{\mathrm{a}}$, C. Rambaud ${ }^{\mathrm{a}}$, S. Longeville ${ }^{\mathrm{b}}$ and M. Plazanet ${ }^{\mathrm{a}, \mathrm{c} *}$ \\ ${ }^{a}$ LIPhy, University of Grenoble-Alpes and CNRS - UMR 5588, Grenoble, France \\ ${ }^{b}$ Laboratoire Lon Brillouin, Gif-sur-Yvette, France \\ ${ }^{c}$ Dipartimento di Fisica e Geologia, University of Perugia, Italy
}

(July 2015)

\begin{abstract}
Physical gels formed by amphiphilic molecules, namely in this study Methyl-4,6-Obenzylidene- $\alpha$-D-mannopyranoside, can be form either in polar and protic liquid like water or in organic apolar solvent such as toluene. The solvent, that influences the supramolecular organisation of the gelators, plays an important role in the stability and formation of the gel phase. Gelator-solvent interactions govern not only the assembly but also the solvent diffusion in the material. We present here measurements of neutron scattering (Time of Flight and Neutron Spin Echo) characterising this microscopic behaviour. In addition, we show that Transient Grating spectroscopy provides valuable information through the characterisation of the longitudinal acoustic wave propagating in the system. Opposite effects on the speed of sound in the gels are observed for the two solvents investigated, being relevant of the interactions between the gelators and the surrounding liquid.
\end{abstract}

Keywords: molecular gel ; physical gel ; neutron scattering ; Transient Grating Spectroscopy ; picosecond dynamics ; sound propagation

\section{Introduction}

Organization and properties of self-assembly molecular materials depend on the nature of the surrounding media. This is the case of Low Molecular Mass Organic Gelators, that can form physical gels with a large panel of solvents of different chemical characteristics [1]. For an amphiphilic gelator, gels can be formed in solvents as different as toluene and water. As a model system of this kind, we focused our attention on the Methyl-4,6-O-benzylidene- $\alpha$-D-mannopyranoside ( $\alpha$-manno), that belongs to the family of substituted benzylido-mono-pyranoside [2, 3], and is composed of an aromatic part attached to a mannose molecule. In organic solvents, the gelators self-assemble through hydrogen bonds between sugars and the benzylidene groups are free to rotate in the solvent, while in water the aromatic rings pile thanks to $\pi-\pi$ interactions and the sugars form hydrogen bonds with the surrounding liquid [3]. Such a system presents various interests. From a chemical and applicative point of view, it changes its organisation and properties depending on the environment or external stimuli, entering the class of so-called smart materials. From a more fundamental point of view, the mechanisms of gel formation and the prediction of its stability are not understood. Moreover, this soft material is a nano- 
to micro-porous matrix in which the liquid diffuses, where the matrix formation and stability depends on the gelator-liquid interactions themselves. If liquid transport at the nanoscale in hard matrices such as porous silicate glasses has already been given a large interest[4-6], little is known when the matrix is soft, rearranging due to the interactions between gelators and solvent. In this context, we aim at characterising the behaviour of liquids in such a gel and the interactions between liquid and gelators.

In a previous work [7], we investigated the implication of solvent molecules into the rigid network. We could quantify the number of solvent molecules that participate to the rigid assembly, a number that depends on the gelator part which is solvated. We could then identify two solvent populations, the bulk-like one and the one trapped in the network, but we were not able to distinguish a dynamically intermediate one. Moreover, we were not able to distinguish any relaxation mode of the network.

We present here further investigations of the solvent behaviour on the pico to nanosecond timescales, and its behaviour in temperature across the gel-sol transition. We also report our first results of transient grating spectroscopy which provide, through the measurement of the speed of sound, valuable information on the gelators-liquid interactions.

\section{Material and Methods}

Samples. $\alpha$-manno was bough from Orgentis Chemicals and dried under vacuum, and deuterated toluene from Sigma-Aldrich. Samples were prepared by dissolving the gelators powder in the solvent at $80^{\circ} \mathrm{C}$, the gel forming during the (not controlled) cooling to room temperature. Gels in toluene were prepared under inert atmosphere, gelators were previously dried under vacuum and toluene over molecular sieve, in order to avoid any water contamination that may lead to partial crystallisation [8]. Typical concentration of 10 to $50 \mathrm{~g} / \mathrm{L}$ were used, depending on the sample and the experiment. The sol-gel transition of $\alpha$-manno in water and toluene, although varying with the concentration, is in our cases around $70^{\circ} \mathrm{C}$ in water and $55^{\circ} \mathrm{C}$ in toluene.[9]

Neutron scattering. Time of flight measurements were performed at the Institut Laue Langevin (France) on IN5 [10] with an incident wavelength of $5 \AA$, leading to a FWHM resolution of $100 \mu \mathrm{eV}$ and a $\mathrm{Q}$ range of $[0.2,2.2] \AA^{-1}$. Neutron Spin echo measurements were performed on the spectrometer MUSES at the Laboratoire Léon Brillouin (France) [11] at individual Q values of 1.2, 1.4 and $1.8 \AA^{-1}$, and discrete time values ranging from 0.1 to $160 \mathrm{ps}$. Deuterated water and toluene were used, with protonated gelators.

Transient Grating. The Transient Grating (TG) setup, as used in the present experiment, enables the characterisation of the longitudinal acoustic wave. Two pump pulses of $400 \mathrm{ps}$ at $1064 \mathrm{~nm}$ (Teem Photonics Powerchip 90) interfere on the sample on which they photo-induce a transient refractive index grating, due to slight heat deposition and electrostriction. The probe beam, a monomode longitudinal cw laser at $532 \mathrm{~nm}$ (Cobolt Samba 1W) is diffracted by this transient grating. The diffracted intensity is monitored as a function of time with a $150 \mathrm{MHz}$ Si amplified photodiode (Thorlabs) and a $3.5 \mathrm{GHz}$ Lecroy oscilloscope. The heterodyne detection is achieved thanks to a phase grating that gives the direct condition of phase matching of the 
four necessary beams (two pumps, one signal and one reference). The momentum transfer $\mathbf{Q}=\left|\mathbf{k}_{\mathbf{i}}-\mathbf{k}_{\mathbf{f}}\right|$ at which are measured the relaxations is then given by the period of the photoinduced grating, $Q=0.63 \mu \mathrm{m}^{-1}$ in our setup. The heat deposited in the bright interference fringes creates a density grating, launching two counterpropagating density waves adding into a stationary wave. The diffracted intensity of the probe therefore oscillates with the period of the acoustic wave and the oscillations vanish with the acoustic damping time. Additionally, the electrostrictive effect increases the amplitude of the density wave, with a phase of $\pi$ with respect to the thermal effect. At longer times, the grating eventually vanishes with the thermal diffusivity characteristic time. The data can then be fitted according to the phenomenological equation [12]:

$$
I_{T G}=A \cdot\left(e^{\left(-t / \tau_{t h}\right)}-e^{-t / \tau_{s}}\right) \cdot \cos (\omega t)+B \cdot e^{-t / \tau_{s}} \cdot \sin (\omega t)
$$

were $\omega=c \cdot q$ is the pulsation of the acoustic wave, $q$ the momentum transfer and $c$ the speed of sound in the sample; $\tau_{t h}$ and $\tau_{s}$ are respectively the thermal diffusivity and sound damping time constants ; $A$ and $B$ are respectively the amplitudes of the signal arising from the heat deposition and the electrostrictive effect. In any case, the absorption of the pump beam is not sufficient to induce the melting of the gel.

\section{Results and discussion}

\section{Relaxation dynamics}

Previous investigations based on quasi elastic neutron scattering enabled a quantitative evaluation of the number of solvent molecules trapped in the network formed by gelators, thanks to an analysis of the elastic intensity[7]. In a general way, the elastic intensity in a QENS spectrum is indeed given by the atoms that are immobile on the timescale of the instrumental resolution, plus the contributions that arise from geometrically confined relaxations, like the rotation. The immobile atoms contribute with a Q-independent signal, while atoms undergoing rotational motion contribute with an intensity dropping at high $\mathrm{Q}$. We assume in our system that the molecules participating to the rigid network are translationally immobile, although they may undergo rotational or other large amplitude motions. Rigorously, the elastic peak arises therefore only in presence of the rigid network. However, in an experiment, the width associated with translational dynamics at small Q may become too small to broaden the resolution and the intensity may appear as elastic. Following this hypothesis, our previous analysis [7] showed that in water and toluene, about 4 and 10 solvent molecules, respectively, participate to the elastic intensity, therefore with slower relaxation than $\sim 1.3 \mathrm{~ns}$, a timescale given by the combination of the Time of Flight and Backscattering techniques. We were moreover able to distinguish only two solvent populations: the immobile one, and the one with bulk characteristics.

As a function of temperature, the elastic peak remains a signature of the gel phase, as we show here in the case of $\alpha$-manno in water. The quasi-elastic spectra were fitted by a sum of lorenzian contributions, arising from translational and rotational diffusion, and the elastic contribution arising mainly from immobile atoms. As can be seen on the figure 1 in a $\alpha$-manno-water sample, the elastic intensity slightly decreases between room temperature and $57^{\circ} \mathrm{C}$, and drops at $90^{\circ} \mathrm{C}$, above the gel-sol transition. Similarly to what observed at room temperature, 


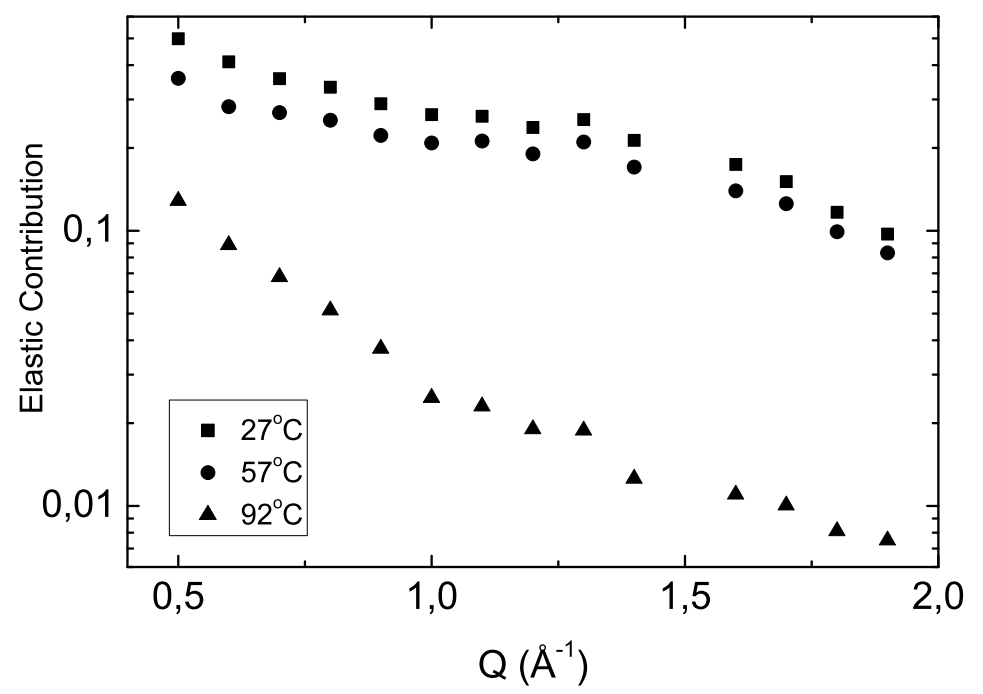

Figure 1. Temperature dependence of the elastic peak intensity in $\alpha$-manno-water, measured on IN5 (ILL).

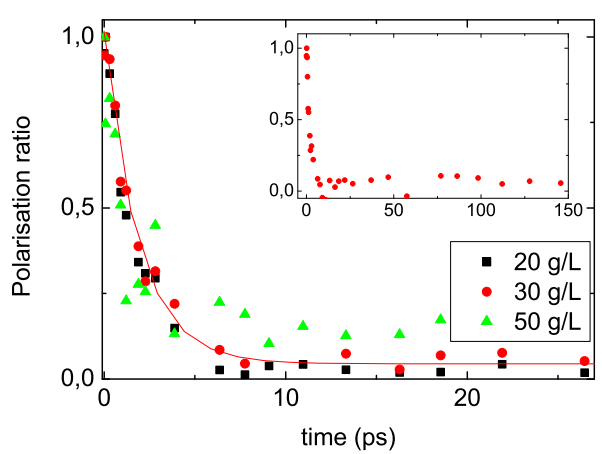

(a) manno-D2O measured at $\mathrm{Q}=1.8 \AA-1$.

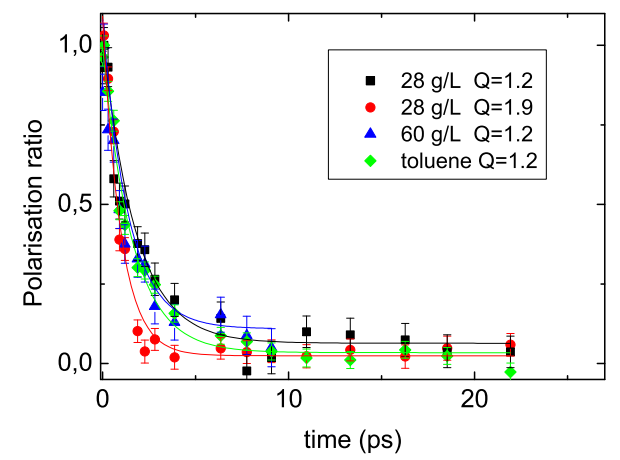

(b) manno-Toluene-d7.

Figure 2. NSE measured on MUSES (LLB) on various gel samples at room temperature.

the rotational and translational contributions present the same characteristics as bulk water (data not shown). No intermediate population is observed, and in the solution, the presence of gelators is not detected in the diffusion coefficient of water.

Neutron spin echo enables to probe the dynamics directly in the time domain, reaching longer times than the previously used techniques. In this experiment, we used protonated gelators in perdeuterated solvents, $\alpha$-manno in toluene-d7 and $\mathrm{D}_{2} \mathrm{O}$, with the aim of characterising an eventual slower solvent population than the one having bulk characteristics, and the dynamics of the self-assembled gelators chains. Regarding the solvent, we would therefore get information on the collective dynamics rather than the self diffusion.

Results are shown in the figure 2. Times were probed up to 150 ps. However, no additional relaxation with respect to previous observations could be identified. The translational diffusion of solvent gives the decrease of polarisation at short times, and no further decay could be observed. Because of instrumental problems during the experiment, we were not able to conclude about the dynamics between 150 ps and $1.4 \mathrm{~ns}$. 


\section{Sound velocity}

Using transient grating spectroscopy, we were able to measure the sound velocity, as extracted from the fitting procedure according to equation (1), as a function of temperature in a mixture of $\alpha$-manno - toluene and $\alpha$-manno - water, and compare it with the pure liquids. The speed of sound in a liquid is related to the ratio between the bulk modulus, or inverse of isothermal compressibility, and the density by the relation $c^{2}=\frac{B}{\rho}=\frac{1}{\chi_{T} \rho}$. In our case, the measurement of the sound velocity therefore gives information on the interactions between solvent and gelators, through the density and rigidity variations with respect to the pure liquid. The results of the evolution of the speed of sound over a temperature range crossing the sol-gel transition are presented in the figure 3 .

In toluene, which behaves like most of the liquids, the sound velocity decreases with temperature, although the density of toluene between 30 and $90^{\circ} \mathrm{C}$ decreases from 857.5 and $799.9 \mathrm{~kg} / \mathrm{m}^{3}$ [13]. This variation would lead to an increase in the sound velocity of $3.5 \%$, while a decrease of $20 \%$ is observed, therefore caused by a decrease of the bulk modulus. In the $\alpha$-manno - toluene gel at $20 \mathrm{~g} / \mathrm{L}$, we observe an increase in the sound velocity of $3.7 \%$ with respect to pure toluene. This difference is strikingly constant over the whole temperature range, in particular in the gel as well as in the solution. The interactions between $\alpha$-manno and toluene therefore lead to an increase of density and/or of the compressibility of the liquid, independently of the assembling of the $\alpha$-manno molecules in the solvent.

Differently, water exhibits an increase of the speed of sound with temperature up to a maxima around $70^{\circ} \mathrm{C}$ because of a minimum in the density and a minimum in the compressibility $[14,15]$. When $\alpha$-manno is mixed to water, at high temperature, a decrease of speed of sound is observed. And unlike in the toluene case, the deviation from pure water is not constant over the whole temperature range but progressively vanishes when temperature decreases.

These observations have to be confronted with the supramolecular organisation of the gelators in both liquids. In water, aromatic rings are piled and hydrogen bonds are formed between water and the sugar moieties, creating a hydration shell around the sugar parts and disturbing the hydrogen bond water network. Upon gelation, the difference between the gel and the pure solvent vanishes. According to previous results, around 10 to 14 water molecules are involved in the rigid structure of the gel. This evolution indicates a reorganisation of the hydrogen bounding between gelators and water during the transition, with a water structure perturbed in the solution and closer to a bulk behaviour in the gel phase.

The gel formed in toluene behaves in the opposite way. The gelators assemble through hydrogen bonds and aromatic parts are solvated by the toluene molecules. The gelators may readily form dimers in the sol phase, leading to more rigid objects in the sample and decreasing the overall compressibility or density. When the temperature decreases, the reduced thermal agitation enables larger network of gelators to form, but in average the compressibility and density of the system do not change.

\section{Conclusions}

We investigated the behaviour of an amphiphile gelator, $\alpha$-manno, in two different liquids, toluene and water. We showed that in a quasi-elastic neutron scattering measurement, the elastic peak provides a signature of the gel phase, without im- 


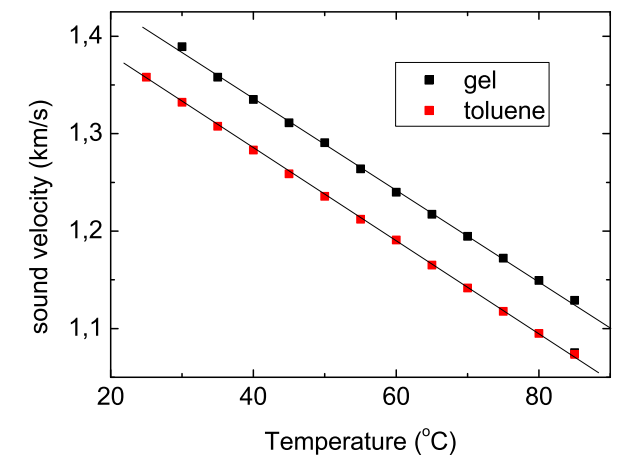

(a) $\alpha$-manno-Toluene (lines are guides for the eyes only).

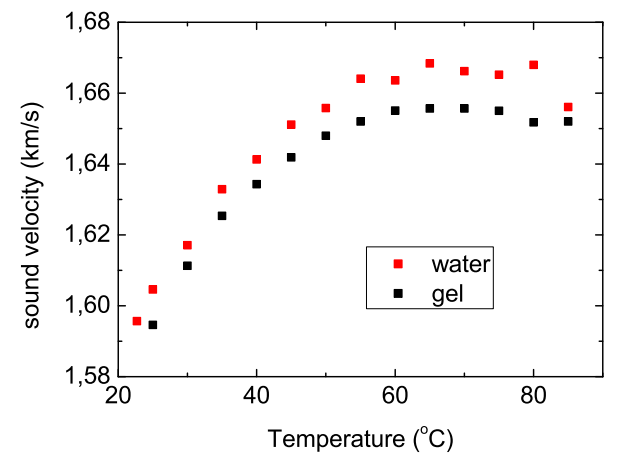

(b) $\alpha$-manno-water.

Figure 3. Sound velocity measured by Transient Grating techniques on both gels and pure liquids as a function of temperature.

portant variation up to the melting of the gelators network. Some solvent molecules are trapped in the network, but their relaxation time could not be measured neither by QENS technique nor by Neutron Spin Echo. The dynamics of the non covalent chains formed by the gelators may be characterised using NSE, but combined with the use of perdeuterated gelators. Simultaneously, Transient Grating proves to be a valuable technique to probe the gelators-solvent interactions, showing opposite behaviour of the gels with respect to the pure liquid when formed in liquid of different properties. More quantitative information will be extracted with the knowledge of density over the whole temperature range.

\section{Acknowledgements}

We thank Michel Bouriau for the help in setting up the TG experiment, and Thomas Derbomez for the help during the TG measurements. We also thank M.A. Gonzalez and J. Ollivier for their assistance during the experiments at the ILL.

\section{References}

[1] P. Terech and R. Weiss, Chem. Rev. 2665 (1997) p. 3133.

[2] O. Gronwald and S. Shinkai, J. Chem. Soc., Perkin Trans. 2 (2001) p. 1933.

[3] O. Gronwald and S. Shinkai, Chem.- Eur. J. 7 (2001) p. 4328.

[4] C. Alba-Simionesco, B. Coasne, G. Dosseh, G. Dudziak, K.E. Gubbins, R. Radhakrishnan and M. Sliwinska-Bartkowiak, Journal of physics. Condensed matter : an Institute of Physics journal 18 (2006) p. R15.

[5] A. Taschin, P. Bartolini, A. Marcelli, R. Righini and R. Torre, Journal of Physics: Condensed Matter 27 (2015) p. 194107.

[6] M. Vogel, Eur. Phys. J.: Spec. Top. 189 (2010) p. 47.

[7] S. Spagnoli, I. Morfin, M.A. Gonzalez, P. Çar,cabal and M. Plazanet, Langmuir 31 (2015) p. 2554.

[8] M. Plazanet, P. Bartolini, C. Sangregorio, A. Taschin, R. Torre and H.p. Trommsdorff, Physical chemistry chemical physics : PCCP 12 (2010) p. 7026.

[9] K. Yoza, N. Amanokura, Y. Ono, T. Akao, H. Shinmori, M. Takeuchi, S. Shinkai and D.N. Reinhoudt, Chem. - Eur. J. 5 (1999) p. 2722.

[10] J. Ollivier, M. Plazanet, H. Schober and J. Cook, Phys. B 350 (2004) p. 173. 
[11] S. Longeville Available at http://www-llb.cea.fr/spectros/pdf/muses-llb.pdf.

[12] Y.X. Yan and K.A. Nelson, J. Chem. Phys. 87 (1987) p. 6240.

[13] M.O. McLinden and J.D. Splett, A liquid density standard over wide ranges of temperature and pressure based on toluene (2008).

[14] C.L. O'Connor, J. Chem. Phys. 47 (1967) p. 31.

[15] A. Taschin, P. Bartolini, M. Ricci and R. Torre, Philosophical Magazine 84 (2004) p. 1471.

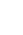

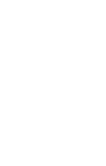

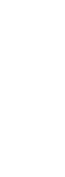

